\title{
Periodic and quasiperiodic motion of an elongated microswimmer in Poiseuille flow
}

\author{
Andreas Zöttla and Holger Stark \\ Institut für Theoretische Physik, Technische Universität Berlin, Hardenbergstrasse 36, 10623 Berlin, Germany
}

Received 1 June 2012 and Received in final form 22 October 2012

Published online: 17 January 2013

(C) The Authors 2013. This article is published with open access at Springerlink.com

\begin{abstract}
We study the dynamics of a prolate spheroidal microswimmer in Poiseuille flow for different flow geometries. When moving between two parallel plates or in a cylindrical microchannel, the swimmer performs either periodic swinging or periodic tumbling motion. Although the trajectories of spherical and elongated swimmers are qualitatively similar, the swinging and tumbling frequency strongly depends on the aspect ratio of the swimmer. In channels with reduced symmetry the swimmers perform quasiperiodic motion which we demonstrate explicitly for swimming in a channel with elliptical cross-section.
\end{abstract}

\section{Introduction}

Swimming on the micron scale has attracted a lot of attention among physicists, since they try to understand the fascinating strategies that microorganisms employ to overcome the constraints of low-Reynolds-number hydrodynamics $[1,2]$. On the other hand, artificial microscopic swimmers or active particles with various locomotion mechanisms have been constructed recently also with the goal of using controllable environments to study the principal properties of their diffusive and collective motion, as well as locomotion under external fields such as gravity or shear flow [3-11]. The experiments are accompanied by detailed theoretical studies analyzing hydrodynamic (see, e.g., [12-20]), stochastic (see, e.g., [21-24]), chemotactic (see, e.g., [25,26]), optical [27] or thermophoretic [28] effects on the dynamics of active particles.

In nature, microorganisms move in an aqueous environment and often have to respond to fluid flow like microscopic plankton in the sea [29], pathogens in the blood stream [30], or sperm cells in the fallopian tubes when swimming towards the egg [31]. Rheotaxis of sperm cells [32] or bacteria [33] allow directed motion along flow gradients. Bottom-heavy microorganisms show stable orientations in flow resulting in layering of phytoplankton in the ocean [34] or hydrodynamic focussing in Poiseuille flow $[35,36]$. Neutrally buoyant microswimmers, however, follow periodic trajectories when swimming in Poiseuille flow $[37,38,30]$. Finally, microswimmers in timedependent flow easily show chaotic motion and non-trivial transport phenomena [39-41].

\footnotetext{
a e-mail: andreas.zoettl@tu-berlin.de
}

In our previous paper we have studied the dynamics of spherical microswimmers in cylindrical Poiseuille flow and identified two basic swimming states, an upstream oriented swinging motion around the centerline and tumbling similar to passive particles [38]. In this article, we extend our work and analyze the motion of elongated microswimmers, as they occur more frequently in nature, subject to Poiseuille flow in microchannels with different geometries. In our study, we will assume that gravity or other external forces are not present or not relevant. Our swimmers do not exhibit active rotation (as in ref. [42]), so their local reorientation in flow is the same as for passive elongated particles. The hydrodynamic reorientation in low-Reynolds-number flow depends on both the local flow vorticity and the local strain rate in the fluid. They both determine the periodic tumbling motion of passive ellipsoidal particles in flow commonly known as Jefferey orbit [43]. The strain rate does not influence the orientation of spherical particles and was therefore not considered in our paper [38].

Now, in contrast to passive particles, microswimmers also change their position in flow due to their active motion. While rigid passive particles just follow streamlines during rotation [44], active particles cross streamlines because of their self-propulsion. In this article we demonstrate that microswimmers with elongated shape still perform the characteristic swinging and tumbling motion in steady Poiseuille flow as observed for spherical swimmers. The motion is periodic in planar Poiseuille flow or in cylindrical tubes with spherical cross-section. Interestingly, the motion becomes quasiperiodic even for spherical swimmers when the flow cross-section becomes elliptical.

In the following we first introduce the equations of motion in sect. 2, discuss the swimmer trajectories for differ- 
(a)

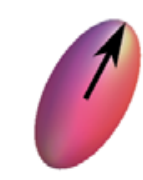

(b)

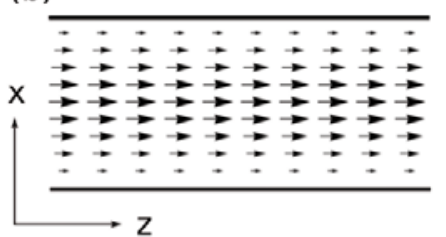

(c)

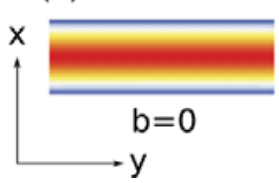

(d)

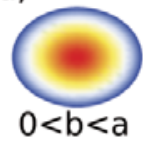

(e)

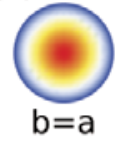

Fig. 1. (a) Spheroidal microswimmer with aspect ratio $\gamma$ and orientation $\boldsymbol{e}$ (black arrow). (b) Sketch of the Poiseuille flow profile in the $x-z$ plane. (c)-(e) Sketch of the channel crosssections ( $x-y$ plane) with semimajor axis $a^{-1}$ and semiminor axis $b^{-1}$ and color-coded flow strength for (c) two parallel and infinitely extended plates $(b=0)$ and a channel with (d) elliptical $(0<b<a)$ and $(\mathrm{e})$ circular $(b=a)$ cross-section.

ent flow cross-sections in sect. 3, and conclude with final remarks in sect. 4 .

\section{Equations of motion}

We study the dynamics of an elongated or prolate spheroidal microswimmer with aspect ratio $\gamma$ in a steady laminar flow $\boldsymbol{v}_{f}(\boldsymbol{r})$ at low Reynolds number. In bulk the swimmer moves with a constant velocity $\boldsymbol{v}_{0}=v_{0} \boldsymbol{e}$, where $\boldsymbol{e}$ is the swimming direction along the major axis of the swimmer (fig. 1(a)). Low Reynolds number also means that we can neglect any inertia of the microswimmer. In addition, we consider the case of large rotational Peclet number $P e_{r}=\tau_{r} / t_{0}$, where $\tau_{r}=1 /\left(2 D_{r}\right)$ is the rotational diffusion time with $D_{r}$ the rotational diffusion constant, and $t_{0}$ the time a swimmer needs for one full oscillation. So $P e_{r}$ describes the number of turns for the swimmer where rotational diffusion can be neglected. Furthermore, the microswimmer should be small compared to the lateral extension of the imposed flow in the microchannel and should not disturb the Poiseuille flow field. Finally, we assume that the swimmer stays away from bounding walls so that we can neglect steric and hydrodynamic interactions between swimmer and walls which otherwise play an important role $[45-48,38]$. Under these conditions, a passive particle simply follows the streamlines with its center-ofmass velocity at position $\boldsymbol{r}$ given by $\boldsymbol{v}(\boldsymbol{r})=\boldsymbol{v}_{f}(\boldsymbol{r})$. Its orientation moves on a Jefferey orbit, where the total angular velocity for a prolate spheroidal particle reads [43]

$$
\boldsymbol{\Omega}(\boldsymbol{r})=\frac{1}{2} \boldsymbol{\Omega}_{f}(\boldsymbol{r})+G \boldsymbol{e} \times[\mathrm{E}(\boldsymbol{r}) \cdot \boldsymbol{e}] .
$$

Here we have introduced the local flow vorticity

$$
\boldsymbol{\Omega}_{f}(\boldsymbol{r})=\nabla \times \boldsymbol{v}_{f}(\boldsymbol{r})
$$

and the local strain rate

$$
\mathrm{E}(\boldsymbol{r})=\frac{1}{2}\left[\nabla \boldsymbol{v}_{f}(\boldsymbol{r})+\left(\nabla \boldsymbol{v}_{f}(\boldsymbol{r})\right)^{T}\right] .
$$

The geometry factor $G=\frac{\gamma^{2}-1}{\gamma^{2}+1}$ with $G \in[0,1)$ depends on the aspect ratio $\gamma$. For spherical swimmers, where $\gamma=1$ and $G=0$, only the flow vorticity $\boldsymbol{\Omega}_{f}$ contributes to the swimmer's angular velocity. An active particle with intrinsic swimming speed $v_{0}$ and moving along its orientation $\boldsymbol{e}$ assumes the total velocity

$$
\boldsymbol{v}(\boldsymbol{r})=\boldsymbol{v}_{f}(\boldsymbol{r})+v_{0} \boldsymbol{e}
$$

so that it can easily cross streamlines. Since we do not assume an intrinsic rotation rate of the swimmer, the angular velocity is the same as for a passive particle (eq. (1)). Using eqs. (1) and (4) the dynamics for position $\boldsymbol{r}(t)$ and orientation $\boldsymbol{e}(t)$ of the swimmer follows

$$
\frac{\mathrm{d}}{\mathrm{d} t} \boldsymbol{r}=\boldsymbol{v}(\boldsymbol{r}), \quad \frac{\mathrm{d}}{\mathrm{d} t} \boldsymbol{e}=\boldsymbol{\Omega}(\boldsymbol{r}) \times \boldsymbol{e} .
$$

We consider a Poiseuille flow along the $z$ direction,

$$
\boldsymbol{v}_{f}(x, y)=v_{f}\left[1-(a x)^{2}-(b y)^{2}\right] \hat{\boldsymbol{z}},
$$

where we use Cartesian coordinates $(x, y, z)$ with the coordinate basis $(\hat{\boldsymbol{x}}, \hat{\boldsymbol{y}}, \hat{\boldsymbol{z}})$ and $v_{f}$ as the maximum flow velocity in the center of the channel. For $b=0$, eq. (6) describes the flow between two parallel and infinitely extended plates. For $b=a$ the flow occurs in a cylindrical channel, and for $0<b<a$ the channel has a general elliptical cross-section, where $a^{-1}$ is the semimajor axis and $b^{-1}$ the semiminor axis (fig. 1(b-e)). The flow vorticity is given by

$$
\boldsymbol{\Omega}_{f}(x, y)=2 v_{f}\left(a^{2} x \hat{\boldsymbol{y}}-b^{2} y \hat{\boldsymbol{x}}\right)
$$

and the strain rate is

$$
\mathrm{E}=-v_{f}\left(a^{2} x \hat{\boldsymbol{x}} \hat{\boldsymbol{z}}+b^{2} y \hat{\boldsymbol{y}} \hat{\boldsymbol{z}}+a^{2} x \hat{\boldsymbol{z}} \hat{\boldsymbol{x}}+b^{2} y \hat{\boldsymbol{z}} \hat{\boldsymbol{y}}\right) .
$$

In the following we use rescaled units, $a x \rightarrow x, b y \rightarrow$ $\bar{b} y, a z \rightarrow z$, and $t / t_{0} \rightarrow t$ with $t_{0}=\left(a v_{0}\right)^{-1}$. We also introduce the dimensionless flow speed $\bar{v}_{f}=v_{f} / v_{0}$ and the aspect ratio of the channel cross-section, $\bar{b}=b / a$. The third parameter of the system is the aspect ratio $\gamma$ of the swimmer.

We note that the general configuration space for the swimmer dynamics is the product $\mathbb{R}^{3} \times \mathbb{S}^{2}$ of position space $\left(\boldsymbol{r} \in \mathbb{R}^{3}\right)$ and unit sphere $\left(\boldsymbol{e} \in \mathbb{S}^{2}\right)$. Since we only consider unidirectional flow, i.e. flow in the $z$-direction, the dynamics of the swimmer does not depend on the $z$ coordinate, and the relevant phase space becomes four-dimensional, $\mathbb{R}^{2} \times \mathbb{S}^{2}$. The dimension of the relevant phase space is further reduced when symmetric flow geometries are used, such as planar or cylindrical flow.

\section{Swimmer dynamics in Poiseuille flow}

In the following we study the deterministic dynamics of a prolate spheroidal microswimmer in Poiseuille flow for several geometries. We start with the motion between two parallel plates $(\bar{b}=0)$, continue with a cylindrical microchannel with circular cross-section $(\bar{b}=1)$, and finally discuss the motion in a channel with general elliptical crosssection $(\bar{b}<1)$. 


\subsection{Planar Poiseuille flow}

First, we discuss the motion of a microswimmer in planar Poiseuille flow, $\boldsymbol{v}_{f}=\bar{v}_{f}\left(1-x^{2}\right) \hat{\boldsymbol{z}}$, with flow vorticity $\boldsymbol{\Omega}_{f}=2 \bar{v}_{f} x \hat{\boldsymbol{y}}$ and strain rate $\mathrm{E}=-\bar{v}_{f} x(\hat{\boldsymbol{x}} \hat{\boldsymbol{z}}+\hat{\boldsymbol{z}} \hat{\boldsymbol{x}})$. For the position of the swimmer we employ Cartesian coordinates, $\boldsymbol{r}=x \hat{\boldsymbol{x}}+y \hat{\boldsymbol{y}}+z \hat{\boldsymbol{z}}$, and we expand the orientation vector $\boldsymbol{e}$ in the Cartesian basis using angular coordiantes $\Psi$ and $\Theta$ such that $\boldsymbol{e}=e_{x}(\Psi, \Theta) \hat{\boldsymbol{x}}+e_{y}(\Theta) \hat{\boldsymbol{y}}+e_{z}(\Psi, \Theta) \hat{\boldsymbol{z}}$, with

$$
e_{x}=-\cos \Theta \sin \Psi, \quad e_{y}=\sin \Theta, \quad e_{z}=-\cos \Theta \cos \Psi .
$$

Here $\Psi$ indicates the orientation relative to the $z$-axis and $\Theta \neq 0$ the orientation out of the $x-z$ plane. We note that $\Psi=0, \Theta=0$ corresponds to perfect upstream orientation and $\Psi=\pi, \Theta=0$ to downstream orientation, respectively. Due to translational symmetry of the flow field in the $y$ direction the relevant configuration space of the swimmer reduces to three dimensions, $\{x, \Psi, \Theta\} \in \mathbb{R} \times \mathbb{S}^{2}$

Using eqs. (5) and (9), the coupled equations of motion for $(x, \Psi, \Theta)$ read

$$
\begin{aligned}
\dot{x} & =-\cos \Theta \sin \Psi, \\
\dot{\Psi} & =\bar{v}_{f} x(1-G \cos 2 \Psi), \\
\dot{\Theta} & =\bar{v}_{f} x \frac{G}{2} \sin 2 \Theta \sin 2 \Psi .
\end{aligned}
$$

Whereas the first equation describes cross-streamline motion of the microswimmer, the second and third equations are the same for passive ellipsoidal particles but now the position $x$ is time-dependent. When the solutions $x(t)$, $\Psi(t)$ and $\Theta(t)$ of eqs. (10) are known, $y(t)$ and $z(t)$ follow by integration

$$
\begin{aligned}
& y(t)=y(0)+\int_{0}^{t} \sin \Theta\left(t^{\prime}\right) \mathrm{d} t^{\prime}, \\
& z(t)=z(0)+\int_{0}^{t}\left[\bar{v}_{f}\left(1-x\left(t^{\prime}\right)^{2}\right)-\cos \Theta\left(t^{\prime}\right) \cos \Psi\left(t^{\prime}\right)\right] \mathrm{d} t^{\prime} .
\end{aligned}
$$

\subsubsection{Two-dimensional motion}

Before discussing the full three-dimensional dynamics of the swimmer, we consider first the two-dimensional case. When $\Theta=0$, the swimmer only moves in the $x$ - $z$-plane and the relevant phase space becomes two-dimensional, $\{x, \Psi\} \in \mathbb{R} \times \mathbb{S}$. The dynamics of position $x$ and orientation $\Psi$ follows from

$$
\begin{aligned}
\dot{\Psi} & =\bar{v}_{f} x(1-G \cos 2 \Psi), \\
\dot{x} & =-\sin \Psi .
\end{aligned}
$$

For a spherical swimmer $(G=0)$ we recover the case treated in ref. [38]. The dynamic equations are $\dot{\Psi}=\bar{v}_{f} x$ and $\dot{x}=-\sin \Psi$, which gives the pendulum equation, $\ddot{\Psi}+$ $\omega_{0}^{2} \sin \Psi=0$, where $x$ plays the role of angular velocity (a)

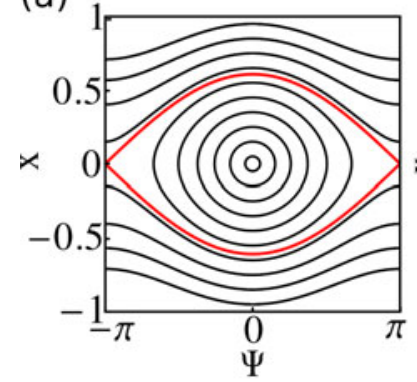

(b)

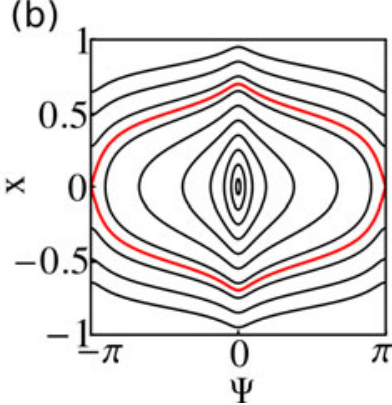

Fig. 2. $\Psi$ - $x$ phase portraits for $\bar{v}_{f}=10$ for a spherical swimmer with $\gamma=1$ or $G=0$ (a) and an elongated swimmer with $\gamma=5$ or $G=0.923$ (b). The red line is the separatrix dividing swinging and tumbling motion.

and $\omega_{0}^{2}=\bar{v}_{f}$. The corresponding constant of motion is the Hamiltonian [38]

$$
H=\frac{1}{2} \bar{v}_{f} x^{2}+1-\cos \Psi .
$$

For elongated swimmers, whose dynamics is governed by eqs. (12), we are also able to identify a constant of motion,

$$
C_{0}=\frac{1}{2} \bar{v}_{f} x^{2}+1-g_{0}(\Psi ; G),
$$

with

$$
g_{0}(\Psi ; G)=\frac{\operatorname{atanh}(\sqrt{2 G /(1+G)} \cos \Psi)}{\sqrt{2 G(1+G)}},
$$

where "atanh" is the inverse function of the hyberbolic tangent. We derive $C_{0}$ in appendix A.1. Note that $C_{0} \rightarrow H$ for $G \rightarrow 0$. For each swimmer trajectory the constant of motion is uniquely defined by the initial conditions $\Psi(0)$ and $x(0)$. We note that steric interactions of the swimmer with the channel wall modify $C_{0}$. After contact with the wall at $|x|=1$ the swimmer leaves the wall with upstream orientation $\Psi=0$ and the constant of motion becomes $C_{0}=\frac{\bar{v}_{f}}{2}+1-g_{0}(0 ; G) \approx \frac{\bar{v}_{f}}{2}+\frac{G}{3}\left(1-\frac{7}{5} G\right)$ for small $G$.

Figure 2 shows the $x-\Psi$ phase portrait for $\bar{v}_{f}=10$. For $G=0$ (or $\gamma=1$ ) it corresponds to the mathematical pendulum (a). The phase portrait for elongated swimmers looks qualitatively the same (b): Closed and open periodic trajectories exist divided by a separatrix. The trajectories are the curves

$$
x^{2}(\Psi)=\frac{2}{\bar{v}_{f}}\left[g_{0}(\Psi ; G)-1+C_{0}\right] .
$$

The closed trajectories correspond to the swinging motion of the microswimmer in Poiseuille flow as discussed in ref. [38]. Since $\Psi=0$ means upstream motion, the swimmer swings around the centerline of the channel while swimming upstream. Because of the upstream orientation, the flow vorticity always reorients the swimmer towards the centerline. After crossing the center at $x=0$ with maximum angle $\Psi_{\max }$, the sign of the vorticity changes 
and the swimmer is reoriented back towards the centerline. So, the swimmer experiences a periodic swinging motion around the centerline, in full analogy to the oscillations of a pendulum. In the channel frame, the swimmer either moves upstream $(\dot{z}<0)$ for sufficiently small flow strengths $\bar{v}_{f}$ whereas it drifts downstream $(\dot{z}>0)$ when $\bar{v}_{f}$ becomes large. Since

$$
\dot{z}(t)=v_{z}(t)+e_{z}(t)=\bar{v}_{f}\left[1-x(t)^{2}\right]-\cos \Psi(t),
$$

the swimmer moves upstream for $v_{z}<-e_{z}$ or $\bar{v}_{f} \lesssim 1$. Perfect upstream swimming in the center of the channel corresponds to the stable fixed point $(\Psi=0, x=0)$.

When the initial state of the swimmer lies outside the separatrix, it tumbles in the flow. The vorticity is too strong for the swimmer to reach the centerline. This is in analogy to the circling motion of a pendulum.

Swinging and tumbling motion are divided by the separatrix (red curves in fig. 2). The trajectories $x(\Psi)$ (eq. (16)) correspond to tumbling when a position $x$ with $\Psi=\pi$ exists or when $g_{0}(\pi ; G)-1+C_{0}>0$. Otherwise they are swinging trajectories. The limiting case is the separatrix which for a fixed $\bar{v}_{f}$ and $G$ is given by

$$
x^{*}\left(\Psi^{*} ; G, \bar{v}_{f}\right)= \pm \sqrt{\frac{2}{\bar{v}_{f}}\left[g_{0}\left(\Psi^{*} ; G\right)-g_{0}(\pi ; G)\right]} .
$$

The separatrix possesses the constant of motion $C_{0}^{*}=$ $1-g_{0}(\pi ; G) \approx 2-\frac{G}{3}\left(1-\frac{7}{5} G\right)$. It includes the spherical swimmer $(G=0)$ with $C_{0}^{*}=H^{*}=2$. For $C_{0}<C_{0}^{*}$ the swimmer is swinging and for $C_{0}>C_{0}^{*}$ it is tumbling.

Linearizing the equations of motion (eqs. (12)) around the fixed point $(\Psi=0, x=0)$ results in a harmonic oscillator equation $\ddot{\Psi}+\bar{v}_{f}(1-G) \Psi=0$ with the eigen-frequency $\omega_{0}=\sqrt{\bar{v}_{f}(1-G)}$. So, elongated swimmers swing with a smaller frequency around the centerline compared to spherical swimmers. The reason is that for elongated swimmers the reorientation rate or angular velocity is smaller compared to spherical swimmers, when $|\Psi|<\pi / 4$. In particular, at fixed $x$ the angular velocity for elongated swimmers (eqs. (12), first line) depends on the orientation, $\dot{\Psi}=\Omega_{y}=\bar{v}_{f} x(1-G \cos 2 \Psi)$, in contrast to spherical swimmers. We plot the angular velocity $\dot{\Psi}$ versus $\Psi$ in fig. 3(a). For spherical swimmers at position $x$ it does not depend on orientation $\Psi$ (orange line) while for ellipsoidal particles $\dot{\Psi}$ varies with $\Psi$ since the strain rate of the flow contributes to the angular velocity. An elongated swimmer oriented parallel to the flow direction $(\Psi=0$ or $\pi)$ rotates with a smaller $\dot{\Psi}$ as a spherical swimmer, while at perpendicular orientation to the flow $(\Psi \approx \pm \pi / 2)$ the angular velocity is larger. In the extreme case of an infinitely thin needle $(G=1)$ (blue curve in fig. 3(a)), the angular velocity vanishes at $\Psi=0$ and $\Psi=\pi$ and the needle just swims along the flow. We also sketch the reorientation rate for spherical and elongated swimmers in fig. 3(b).

The fact that elongated swimmers rotate slower when oriented in flow direction has a noticeable effect on the

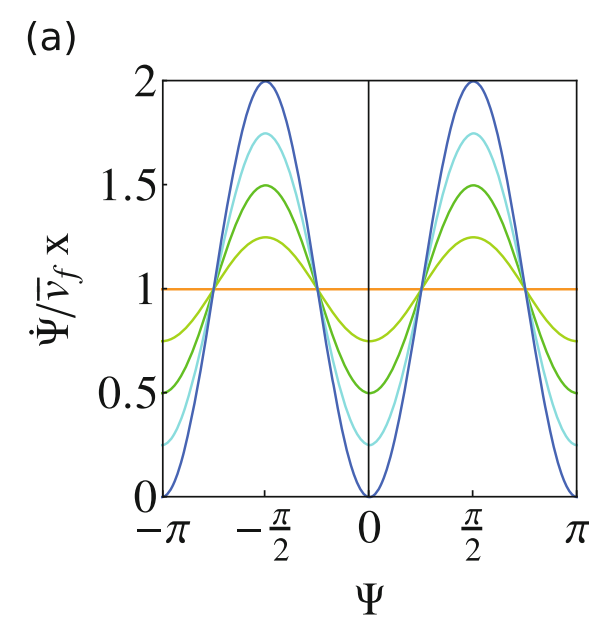

(b)

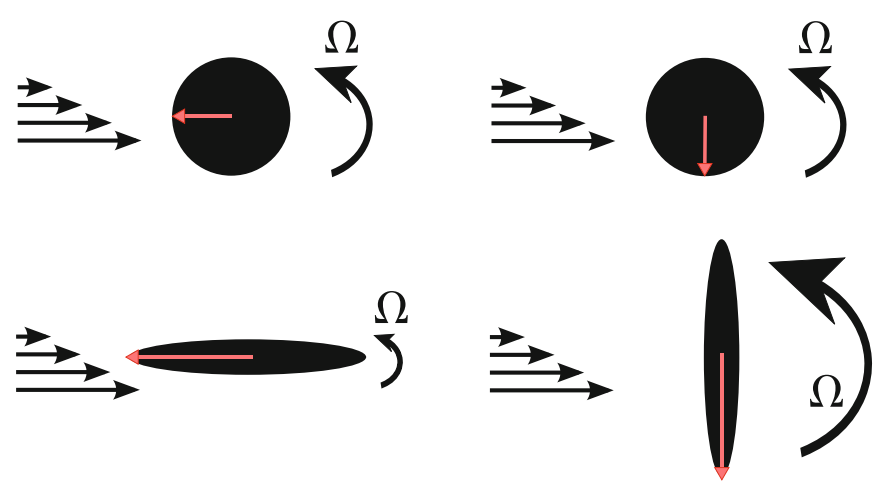

Fig. 3. (a) The angular velocity $\Omega_{y}=\dot{\Psi}$ at position $x$ and given flow strength $\bar{v}_{f}$ plotted versus swimmer orientation $\Psi$ for several shape parameters $G$; orange line: $G=0$; light green: $G=0.25$; dark green: $G=0.5$; light blue: $G=0.75$; dark blue: $G=1$. (b): Sketch of the angular velocities of spherical and elongated swimmers oriented parallel and perpendicular to the flow.

orientational distribution function of the swimmer,

$$
\begin{aligned}
P(\Psi) & \propto|\dot{\Psi}|^{-1} \\
& \propto(1-G \cos 2 \Psi)^{-1}\left(C_{0}-1+g_{0}(\Psi, G)\right)^{-1 / 2} .
\end{aligned}
$$

To arrive at eq. (19), we have eliminated location $x$ in the first line of eqs. (12) using the constant of motion $C_{0}$ of eq. (14). In figs. 4(a) and (b) we show the respective probability distributions for spherical and ellipsoidal swimmers. Spherical swimmers have the same probability distribution as the mathematical pendulum [49]. $P(\Psi)$ assumes its absolute minimum where $\dot{\Psi}$ is largest. This occurs at $\Psi=0$ when the swimmer has its maximum distance $|x|$ from the centerline (see eqs. (12)). In the swinging motion $P(\Psi)$ diverges at the centerline, where $\dot{\Psi}=0$ (green and red curve in fig. 4(a)). For the tumbling motion it stays finite since the swimmer does not reach the centerline (blue curve in fig. 4(a)). For ellipsoidal swimmers $\dot{\Psi}$ also depends on the orientation $\Psi$. Since they rotate slower at $\Psi=0$ 
(a)

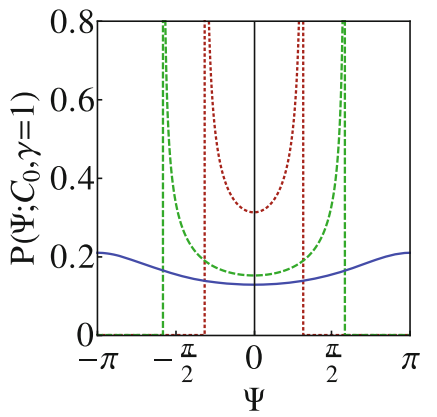

(b)

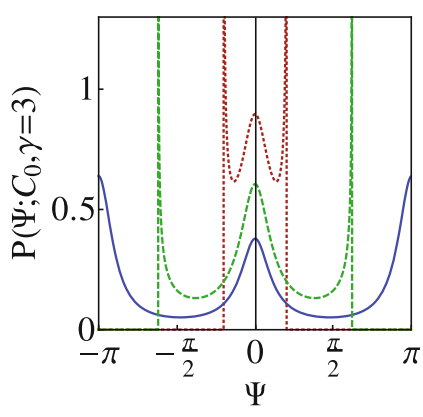

(c)

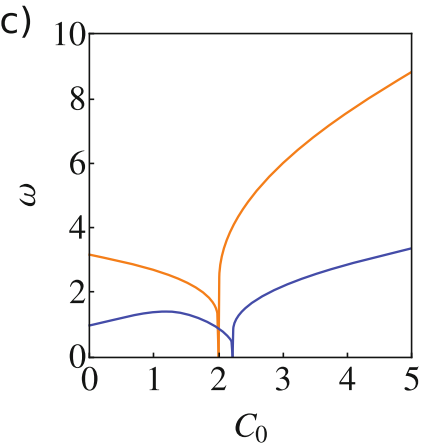

Fig. 4. (a)-(b) Probability distributions $P(\Psi)$ for single trajectories with initial conditions $\Psi(0)=0$ and $x(0)=0.3$ (red), $x(0)=0.5$ (green) and $x(0)=0.8$ (blue) for spherical (a) and elongated (b) particles with $\gamma=3(G=0.8)$. (c) Average angular frequencies $\omega$ for swinging and tumbling motion for $\bar{v}_{f}=10$ for a spherical swimmer (orange) and an elongated swimmer with $\gamma=5(G=0.923)$ (blue) plotted versus the constant of motion $C_{0}$. The frequency for small oscillations is $\omega \approx \sqrt{\bar{v}_{f}(1-G)}$.

when they are oriented parallel to the flow, an additional maximum at $\Psi=0$ appears (fig. 4 (b)).

The reorientation rate $\dot{\Psi}$ also determines the average angular frequency of the swinging and tumbling motion,

$$
\omega=\pi\left(\int_{\Psi_{\min }}^{\Psi_{\max }} \frac{\mathrm{d} \Psi}{|\dot{\Psi}|}\right)^{-1}
$$

For swinging motion $\Psi_{\min }$ and $\Psi_{\max }$ appear when $x=0$, so

$$
\begin{aligned}
& \Psi_{\max }=-\Psi_{\min }= \\
& \operatorname{acos}\left[-\sqrt{(1+G) / 2 G} \tanh \left(\left(C_{0}-1\right) \sqrt{2 G(1+G)}\right)\right],
\end{aligned}
$$

where "acos" is the arccosine and "tanh" the hyperbolic tangent. For tumbling trajectories $\Psi_{\min }=0$ and $\Psi_{\max }=\pi$. We numerically integrate eq. (20) for several geometry factors $G$ and plot it versus $C_{0}$ in fig. 4(c). For spherical swimmers the average frequency $\omega$ decreases for larger oscillations or larger $C_{0}$ in the swinging state in full analogy to the mathematical pendulum (orange curve in fig. 4(c)). Larger oscillations mean that the swimmer orients more

towards a perpendicular orientation relative to the flow direction. Since for elongated swimmers the reorientation rate is largest at $|\Psi|=\pi / 2$, the average frequency $\omega$ first increases until it reaches a maximum for a specific swinging state determined by a specific value of $C_{0}$ (blue curve in fig. 4(c)). In the tumbling state $\omega$ increases with $C_{0}$ for both the spherical and elongated swimmer.

\subsubsection{Three-dimensional motion}

For the three-dimensional motion (eqs. (10)) we are able to identify two constants of motion (see appendix A.2),

$$
\begin{aligned}
& \kappa_{1}=\tan ^{2} \Theta /(1-G \cos 2 \Psi), \\
& \kappa_{2}=\frac{1}{2} \bar{v}_{f} x^{2}+1-g_{1}(\Psi, \Theta ; G),
\end{aligned}
$$

with

$$
g_{1}(\Psi, \Theta ; G)=\frac{\operatorname{atanh}(\sqrt{2 G /(1+G)} \cos \Psi \cos \Theta)}{\sqrt{2 G(1+G)}} .
$$

The intersection of both constants $\kappa_{1}$ and $\kappa_{2}$ in the $x$ $\Psi-\Theta$ phase space defines again periodic solutions of the ellipsoidal swimmer in the planar Poiseuille flow. For $\Theta=$ $0, \kappa_{1}$ vanishes and $\kappa_{2}$ gives $C_{0}$. We thus recover the twodimensional motion discussed in the previous section. In the full three-dimensional case, we can again divide the periodic solutions into swinging and tumbling motions.

Figure 5 illustrates specific examples for $\bar{v}_{f}=10$ and $G=0.8$. Figure $5(\mathrm{a})$ shows the phase space curve for a typical swinging motion and fig. 5(d) for the swimmer in a tumbling state. The initial conditions are $\Psi(0)=0$ and $\Theta(0)=\pi / 5$ for both cases, and $x(0)=0.3$ for (a) and $x(0)=0.7$ for $(\mathrm{d})$. So the constant of motion $\kappa_{1}$ is the same for both cases and it is illustrated by the orange surfaces. The second constant of motion $\kappa_{2}$ (green surfaces) depends on $x$ and thereby defines if swinging or tumbling occurs. The intersection between $\kappa_{1}$ and $\kappa_{2}$ is the actual trajectory in phase space (blue curve). Figures 5(b) and (e) show the motion of the orientation vector $\boldsymbol{e}(t)$. Since it is a unit vector, its arrowhead moves on the unit sphere $\mathbb{S}^{2}$. For the swinging motion $\boldsymbol{e}$ moves back and forth with $e_{y}>0$ on a trajectory that resembles a half-ellipse (b), while it circles around a closed trajectory during tumbling (e). The closed trajectory is similar to the Jefferey orbit of tumbling passive particles [43], however for active particles $x$ is not constant. In fig. $5(\mathrm{c})$ and (f) the trajectories $\boldsymbol{r}(t)$ are shown. For swinging motion the middle plane $(x=0)$ is crossed periodically (c), while tumbling motion always occurs on either side of the middle plane (f). Both trajectories look two-dimensional tilted against the $z$-direction. Since the component $e_{y}(t)$ oscillates with time, they are clearly three-dimensional.

However, for spherical swimmers $G=0$ and $\dot{\Theta}=0$ (eq. (10)), so that the component $e_{y}=\sin \Theta$ is constant in time. The equation of motion for $\Psi$ is again the pendulum equation but it depends on the initial orientation $e_{y}=$ const,

$$
\ddot{\Psi}+\xi \bar{v}_{f} \sin \Psi=0,
$$



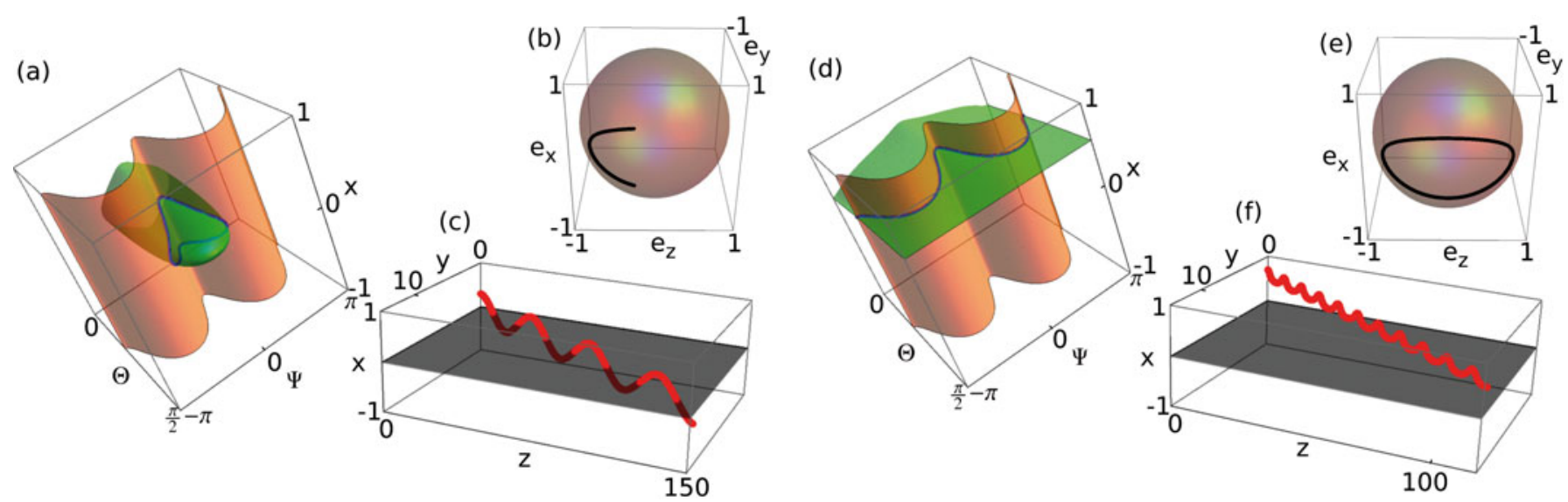

Fig. 5. Swinging (a-c) and tumbling (d-f) motion of a spheroidal swimmer in flow between two parallel plates. The flow strength is $\bar{v}_{f}=10$ and the aspect ratio of the swimmer is $\gamma=3$ (meaning $G=0.8$ ). The initial conditions are $\Psi(0)=0$ and $\Theta(0)=\pi / 5$ for both cases and $x(0)=0.3$ for swinging and $x(0)=0.7$ for tumbling motion. (a,d) Relevant three-dimensional phase space $(x, \Psi, \Theta)$. The intersection of the two constants of motion $\kappa_{1}$ (orange) and $\kappa_{2}$ (green) gives the periodic trajectory (blue intersection curve). While $\kappa_{1}=2.64$ is the same for swinging (a) and tumbling (d), $\kappa_{2}=1.14$ for swinging and $\kappa_{2}=2.86$ for tumbling motion. (b,e) Periodic orbits of the orientation vector $\boldsymbol{e}(t)$ which moves on the unit sphere. For swinging motion it is a half-ellipse (b) and for tumbling motion a closed curve (e). (c,f) Swimmer trajectory $\boldsymbol{r}(t)$ in real space for swinging (c) and tumbling (f). The midplane is shown in grey and the trajectories are red.

where $\xi=\sqrt{1-e_{y}^{2}}=$ const is the length of the orientation vector in the $x$ - $z$ plane. A swimmer starting with $e_{y} \neq 0$ swings or tumbles at smaller frequency $\omega_{0}=\sqrt{\xi \bar{v}_{f}}$ compared to a swimmer with $e_{y}=0$ on a two-dimensional trajectory tilted against the $z$-direction. So, the position $y(t)$ increases linearly in the positive direction for $\Theta(0)>0$ or in the negative direction for $\Theta(0)<0$. Since $\Theta=$ const the relevant phase space is the $x-\Psi$ space similar to the two-dimensional problem.

\subsection{Swimming in a cylindrical tube}

Now we discuss the motion of a swimmer in a cylindrical microchannel. We use cylindrical coordinates $(\rho, \varphi, z)$ and express the orientation vector $\boldsymbol{e}$ in the cylindrical coordinate basis $(\hat{\boldsymbol{\rho}}, \hat{\boldsymbol{\varphi}}, \hat{\boldsymbol{z}}), \hat{\boldsymbol{e}}(\Psi, \Theta)=e_{\rho}(\Psi, \Theta) \hat{\boldsymbol{\rho}}+e_{\varphi}(\Theta) \hat{\boldsymbol{\varphi}}+$ $e_{z}(\Psi, \Theta) \hat{z}$ with

$e_{\rho}=-\cos \Theta \sin \Psi, \quad e_{\varphi}=\sin \Theta, \quad e_{z}=-\cos \Theta \cos \Psi$.

Because of translational symmetry in the $z$-direction and rotational symmetry with respect to $\varphi$, only the equations for $\rho, \Psi$ and $\Theta$ are coupled

$$
\begin{aligned}
\dot{\rho} & =-\cos \Theta \sin \Psi, \\
\dot{\Psi} & =\bar{v}_{f} \rho[1-G \cos 2 \Psi]-\sin \Theta \tan \Theta \cos \Psi / \rho, \\
\dot{\Theta} & =\left(1+2 G \bar{v}_{f} \rho^{2} \cos \Theta \cos \Psi\right) \sin \Theta \sin \Psi / \rho .
\end{aligned}
$$

The relevant phase space $\mathbb{R}^{+} \times \mathbb{S}^{2}$ is again threedimensional. For the spherical microswimmer $(G=0)$ we already identified two constants of motion. They lead to periodic trajectories with helical or helical-like swinging and tumbling motion [38]. We derive the constants of motion in appendix A.3. We were not able to construct constants of motion for an elongated swimmer in cylindrical

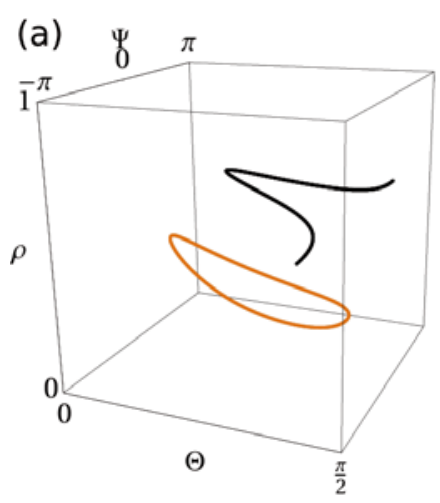

(b)

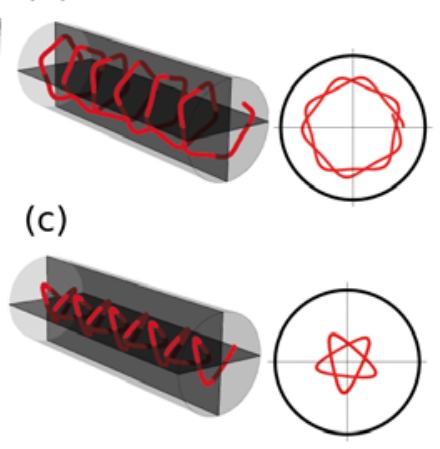

Fig. 6. Swinging and tumbling motion for a microswimmer in a cylindrical tube for the flow strength $\bar{v}_{f}=10$ and swimmer aspect ratio $\gamma=2(G=0.6)$. The initial conditions are $\Psi(0)=\pi / 4, \rho(0)=0.3$ and $\Theta(0)=\pi / 5$ for swinging motion and $\Psi(0)=0, \rho(0)=0.7$ and $\Theta(0)=\pi / 5$ for tumbling. (a) Typical trajectories for swinging (orange) and tumbling (black) motion in the $\rho-\Psi-\Theta$ phase space. The trajectories $\boldsymbol{r}(t)$ in real space are shown in (b) for tumbling motion and in (c) for swinging motion together with projections onto the channel cross-section. Note that the $z$-direction is squeezed relative to the lateral dimension.

Poiseuille flow. Nevertheless, we numerically find that it performs again periodic motions similar to the spherical swimmer. Figure 6(a) shows two closed phase space orbits, both for the swinging and tumbling state, for a swimmer with aspect ratio $\gamma=2$ or geometry factor $G=0.6$. We also illustrate the three-dimensional trajectories in the tube and their two-dimensional projections onto the channel cross-section, figs. 6(b)-(c). For the swinging motion we have chosen initial conditions such that the projection onto the cross-sectional plane is periodic. 
(a)

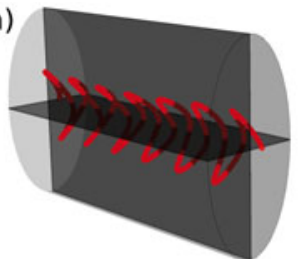

(d)

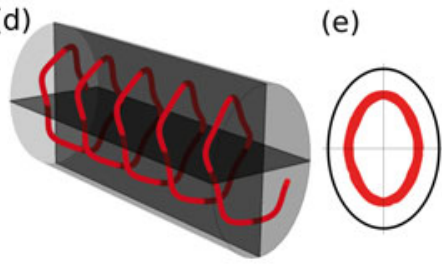

(b)

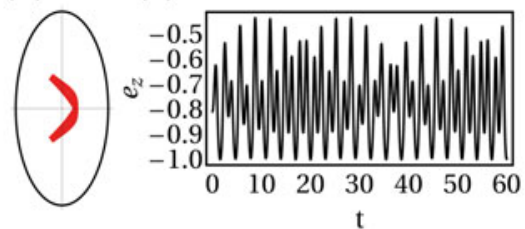

(f)

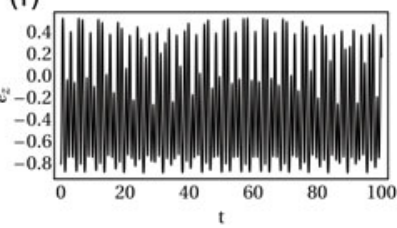

Fig. 7. (a)-(c) Two-frequency quasiperiodic swinging motion for the initial conditions $\Psi(0)=0, \varphi(0)=0, \rho(0)=0.3$, $\Theta(0)=\pi / 5$ and parameters $\bar{b}=2, \gamma=2, \bar{v}_{f}=10$. (d)-(f) Tumbling motion for the initial conditions $\Psi(0)=0, \varphi(0)=$ $0, \rho(0)=0.7, \Theta(0)=\pi / 5$ and parameters $\bar{b}=1.4, \gamma=2$ and $\bar{v}_{f}=10$. (a) and (d): Three-dimensional motion in the elliptical channel. (b) and (e): Trajectories projected onto the cross-section of the channel. (c) and (f): Time evolution of the swimmer orientation component $e_{z}(t)$.

\subsection{Quasiperiodic motion in a channel with elliptical cross-section}

Now we consider the motion in a channel with elliptical cross-section $(\bar{b}<1)$. We again use a cylindrical coordinate system as for the cylindrical channel. The symmetry in $\varphi$ direction no longer exists and the relevant phase space is four-dimensional, $(\rho, \Psi, \Theta, \varphi) \in \mathbb{R}^{2} \times \mathbb{S}^{2}$. The equations of motion for the relevant coordinates read

$$
\begin{aligned}
\dot{\rho}= & -\cos \Theta \sin \Psi, \\
\dot{\Psi}= & \bar{v}_{f} \rho(1-G \cos 2 \Psi)\left[\frac{\bar{b}^{2}+1}{2}+\frac{1-\bar{b}^{2}}{2} \cos 2 \varphi\right] \\
& -\sin \Theta \tan \Theta \cos \Psi / \rho \\
& +\bar{v}_{f} \rho\left(\frac{1-\bar{b}^{2}}{2}\right)(1+G) \sin 2 \varphi \sin \Psi \tan \Theta, \\
\dot{\Theta}= & \frac{\sin \Theta \sin \Psi}{\rho}\left[1+2 G \bar{v}_{f} \rho^{2} \cos \Theta \cos \Psi\left(\cos ^{2} \varphi+\bar{b}^{2} \sin ^{2} \varphi\right)\right] \\
& +\bar{v}_{f} \rho\left(\frac{1-\bar{b}^{2}}{2}\right)(1-G \cos 2 \Theta) \cos \Psi \sin 2 \varphi, \\
\dot{\varphi}= & \sin \Theta / \rho .
\end{aligned}
$$

We again solve them numerically and determine the swimmer trajectories. Interestingly, both spherical and elongated swimmers now follow quasiperiodic trajectories. Figure 7 shows trajectories for a quasiperiodic swinging (fig. 7(a)) and tumbling motion (fig. 7(d)) and their projections onto the elliptical channel cross-sections (figs. 7(b) and (e)). We also show the time evolution of the $z$ component of the orientation vector, $e_{z}(t)$, which shows twofrequency quasiperiodic behavior (fig. 7(c) and (f)) as we

will also demonstrate in fig. 8 . This indicates that the dynamics of a single trajectory takes place on a torus in the four-dimensional phase space. In fig. 8 we further illustrate the complex dynamics of the quasiperiodic swinging (fig. 8(a)-(d)) and tumbling (fig. 8(e)-(h)) dynamics for the same parameters as in fig. 7. Since the quasiperiodic trajectories lie on a torus, they define a two-dimensional surface when projected onto the three-dimensional $\rho-\Psi-\Theta$ subspace of the full phase space. Figures 8(a) and (e) illustrate these complex surfaces. In addition, we plot their Poincaré sections for $\varphi=0, \dot{\varphi}>0$ in fig. 8(c) and (g). The resulting closed curves confirm the two-frequency quasiperiodic motion on a torus [50]. In fig. 8(b) and (f) we show the surfaces again with the azimuthal angle $\varphi$ colorcoded. For the swinging motion (fig. 8(b)) one can clearly identify specific regions in the $\rho-\Psi-\Theta$ space for each value of $\varphi$. The projection of the surface onto the $\rho-\Psi$ plane (see fig. 8(d)) resembles the closed trajectories of the swinging motion discussed earlier but now the curve is smeared out in the $\rho-\Psi$ plane due to the quasiperiodicity. The same is true for the tumbling motion, which we illustrate further in fig. 8(f) and (h). The projected phase space trajectory in the $\rho-\Psi$ plane is a smeared-out version of previous examples (fig. 8(h)). However, the azimuthal angle $\varphi$ in the color-coded surface of fig. $8(\mathrm{f})$ is distributed over the whole phase-space trajectory.

Finally we note that we also studied the dynamics of spherical swimmers which also turned out to be quasiperiodic.

\section{Conclusion}

To conclude, we studied the two- and three-dimensional deterministic dynamics of elongated swimmers in Poiseuille flow at low Reynolds number generalizing our work from ref. [38]. The state of the system is given by five variables, three for the position and two for the orientation in the channel, respectively. For all swimmers we find the same characteristic swimming states. Depending on initial conditions and parameters - aspect ratio of the swimmer $(\gamma)$, geometry of the channel cross-section $(\bar{b})$, and flow strength $\left(\bar{v}_{f}\right)$ - the microswimmer either swings with upstream orientation around the centerline of the channel or it tumbles at sufficiently large flow vorticities. The frequency of swinging and tumbling motion depends on the aspect ratio, in particular, it is smaller for elongated compared to spherical swimmers. For the motion between two infinitely extended parallel plates and for the motion in a cylindrical channel the relevant phase space is only three-dimensional due to the symmetry of the channel cross-section. In these cases we were able to identify two constants of motion which results in a periodic motion visible in the three-dimensional phase space. Interestingly, when we reduce the symmetry by choosing a channel with elliptical cross-section, the phase space becomes four-dimensional and quasiperiodic motion occurs.

Note that in the framework of dynamical systems the motion of swimmers in Poiseuille flow is not considered as dissipative. In laminar flow non-interacting swimmers 
(a)

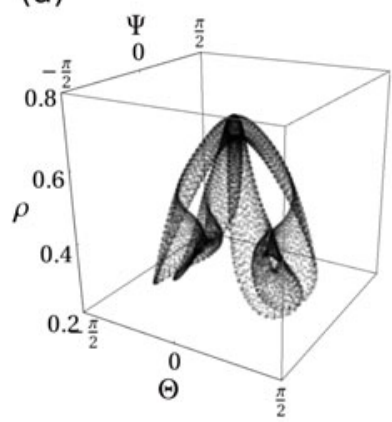

(e)

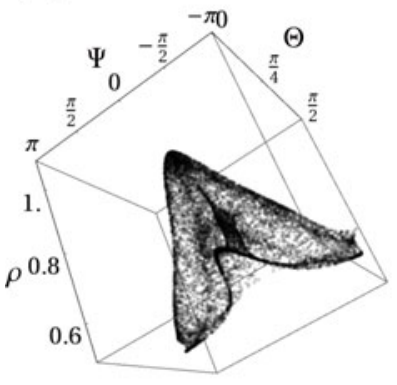

(b)

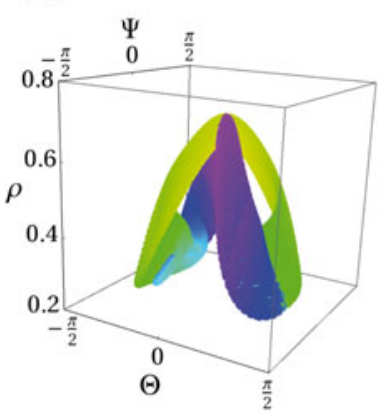

(f)

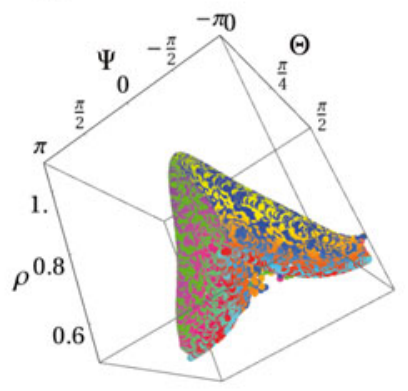

(c)

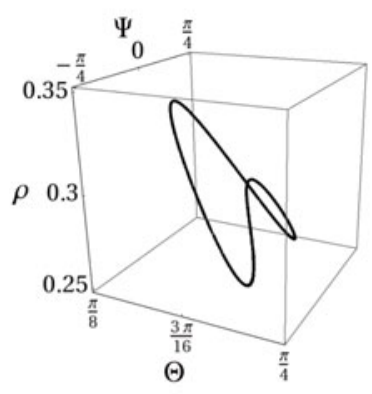

(g)

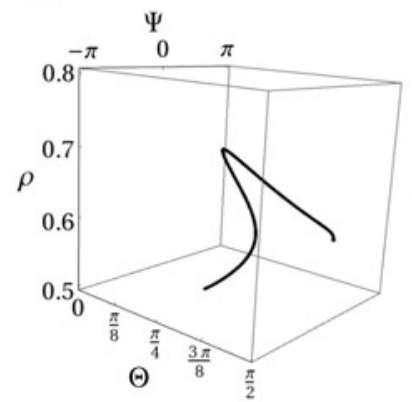

(d)

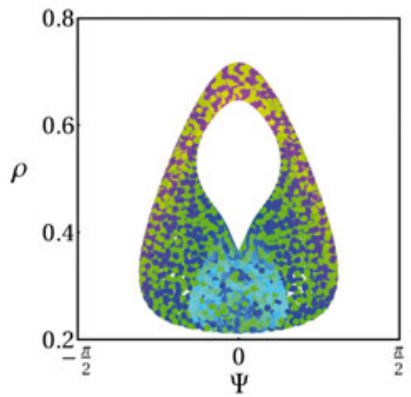

(h)

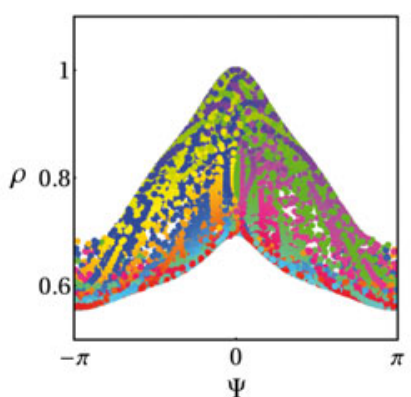

Fig. 8. Projected phase space and Poincaré sections for quasiperiodic swinging (a)-(d) and tumbling (e)-(h) motion. The initial conditions and system parameters are the same as in fig. 7. The projections of the trajectories onto the $\rho-\Psi-\Theta$ phase space lie on two-dimensional surfaces for swinging (a) and tumbling (e) motion. (b) and (f): same trajectories as in (a) and (e) but with the coordinate $\varphi$ color-coded. (c) and $(\mathrm{g})$ : Poincaré sections at $\varphi=0(\dot{\varphi}>0)$. One-frequency behavior in the Poincaré sections indicate two-frequency motion in phase space [50]. (d) and (h): Projections onto the $\rho-\Psi$ phase space where $\varphi$ is color-coded.

move on separate trajectories without aggregating, their dynamics does not reach a common fixpoint or limit cycle. This changes when hydrodynamic interactions between a swimmer and the channel wall play a significant role as demonstrated in ref. [38] where we explicitely identified fixpoints and limit cycles for the spherical swimmer. Other examples are external forces such as gravitation $[34,35,51]$ or external light sources [52] that influence the reorientation of the microswimmers.

This work was supported by the Deutsche Forschungsgemeinschaft within the Research Training Group GRK 1558.

\section{Appendix A. Constants of motion}

\section{Appendix A.1. 2D planar flow}

A constant of motion can be found by dividing the first equation by the second equation of eqs. (12),

$$
\frac{\dot{x}}{\dot{\Psi}}=\frac{\mathrm{d} x}{\mathrm{~d} \Psi}=-\frac{\sin \Psi}{\bar{v}_{f} x(1-G \cos 2 \Psi)} .
$$

This expression can be separated and integrated

$$
\bar{v}_{f} \int x \mathrm{~d} x=-\int \frac{\sin \Psi \mathrm{d} \Psi}{1-G \cos 2 \Psi},
$$

leading to

$$
\frac{\bar{v}_{f}}{2} x^{2}=\frac{\operatorname{atanh}(\sqrt{2 G /(1+G)} \cos \Psi)}{\sqrt{2 G(1+G)}}+c,
$$

where $c$ is a constant and $C_{0}=c+1$ is the constant of motion defined in eq. (14).

\section{Appendix A.2. 3D planar flow}

The first constant of motion $\kappa_{1}$ is identified by calculating

$$
\frac{\dot{\Theta}}{\dot{\Psi}}=\frac{\mathrm{d} \Theta}{\mathrm{d} \Psi}=\frac{G}{2} \frac{\sin 2 \Theta \sin 2 \Psi}{1-G \cos 2 \Psi} .
$$

We again separate the variables and integrate

$$
\int \frac{2 \mathrm{~d} \Theta}{G \sin 2 \Theta}=\int \frac{\sin 2 \Psi \mathrm{d} \Psi}{1-G \cos 2 \Psi},
$$

resulting in

$$
\frac{1}{2 G}[\log (1-G \cos 2 \Psi)]+c=\frac{1}{G}[\log (\tan \Theta)] .
$$

Taking the exponential of this expression and defining $\kappa_{1}$ as $\kappa_{1}=e^{2 G c}$ results in the first constant of motion in 
eqs. (22). Using $\kappa_{1}$, eliminating $\Theta$ and calculating

$$
\frac{\dot{\Psi}}{\dot{x}}=\frac{\mathrm{d} \Psi}{\mathrm{d} x}=-\frac{\bar{v}_{f} x}{\sin \Psi}(1-G \cos 2 \Psi) \sqrt{1+\kappa_{1}(1-G \cos 2 \Psi)},
$$

after separation of variables and integration then leads to

$$
\frac{\bar{v}_{f}}{2} x^{2}=\frac{\operatorname{atanh}(\sqrt{2 G /(1+G)} \cos \Psi \cos \Theta)}{\sqrt{2 G(1+G)}}+c,
$$

where we defined $\kappa_{2}$ as $\kappa_{2}=1+c$ as the second constant of motion in eqs. (22).

\section{Appendix A.3. Cylindrical flow for $G=0$}

The equations of motion for spherical particles swimming in a cylindrical Poiseuille flow are given by eqs. (26) but with $G=0$. Using eqs. (26) the first constant of motion for a spherical swimmer is determined by taking

$$
\frac{\dot{\Theta}}{\dot{\rho}}=\frac{\mathrm{d} \Theta}{\mathrm{d} \rho}=-\tan \Theta / \rho,
$$

after separation of variables and integration leading to

$$
-\log (\sin \Theta)=\log (\rho)+c
$$

and to the first constant of motion $L=e^{-c}=\rho \sin \Theta$ which is proportional to the angular momentum of the swimmer in the $z$-direction. The second constant of motion is identified by taking

$$
\frac{\dot{\Psi}}{\dot{\Theta}}=\frac{\mathrm{d} \Psi}{\mathrm{d} \Theta}=\frac{\bar{v}_{f} L^{2}}{\sin ^{3} \Theta \sin \Psi}-\frac{\tan \Theta}{\tan \Psi} .
$$

This nonlinear differential equation can be transformed into a linear equation by using the ansatz $w=\cos \Psi$ leading to

$$
\frac{\mathrm{d} w}{\mathrm{~d} \Theta}+w \tan \Theta=-\frac{\bar{v}_{f} L^{2}}{\sin ^{3} \Theta} .
$$

The solution is

$$
w=\cos \Psi=\frac{L^{2} \bar{v}_{f}}{2 \sin \Theta \tan \Theta}-\frac{c}{\cos \Theta}
$$

and we define the second constant of motion as

$$
M=c+\frac{1}{2} L^{2} \bar{v}_{f}+1=\frac{1}{2} \bar{v}_{f} \rho^{2}+1-\cos \Theta \cos \Psi .
$$

Open Access This is an open access article distributed under the terms of the Creative Commons Attribution License (http://creativecommons.org/licenses/by/3.0), which permits unrestricted use, distribution, and reproduction in any medium, provided the original work is properly cited.

\section{References}

1. E.M. Purcell, Am. J. Phys. 45, 3 (1977).

2. E. Lauga, T.R. Powers, Rep. Prog. Phys. 72, 096601 (2009).

3. W.F. Paxton, K.C. Kistler, C.C. Olmeda, A. Sen, S.K.St. Angelo, Y. Cao, T.E. Mallouk, P.E. Lammert, V.H. Cresp, J. Am. Chem. Soc. 126, 13424 (2004).

4. R. Dreyfus, J. Baudry, M.L. Roper, M. Fermigier, H.A. Stone, J. Bibette, Nature 437, 862 (2005).

5. E. Gauger, H. Stark, Phys. Rev. E 74, 021907 (2006).

6. J.R. Howse, R.A.L. Jones, A.J. Ryan, T. Gough, R. Vafabakhsh, R. Golestanian, Phys. Rev. Lett. 99, 048102 (2007).

7. H.-R. Jiang, N. Yoshinaga, M. Sano, Phys. Rev. Lett. 105, 268302 (2010).

8. J. Palacci, C. Cottin-Bizonne, C. Ybert, L. Bocquet, Phys. Rev. Lett. 105, 088304 (2010).

9. S. Thutupalli, R. Seemann, S. Herminghaus, New J. Phys. 13, 073021 (2011).

10. G. Volpe, I. Buttinoni, D. Vogt, H.-J. Kümmerer, C. Bechinger, Soft Matter 7, 8810 (2011).

11. I. Theurkauff, C. Cottin-Bizonne, J. Palacci, C. Ybert, L. Bocquet, Phys. Rev. Lett. 108, 268303 (2012).

12. R.A. Simha, S. Ramaswamy, Phys. Rev. Lett. 89, 058101 (2002).

13. Y. Hatwalne, S. Ramaswamy, M. Rao, R.A. Simha, Phys. Rev. Lett. 92, 118101 (2004).

14. J.P. Hernandez-Ortiz, C.G. Stoltz, M.D. Graham, Phys. Rev. Lett. 95, 204501 (2005).

15. I. Llopis, I. Pagonabarraga, EPL 75, 999 (2006).

16. D. Saintillan, M.J. Shelley, Phys. Rev. Lett. 99, 058102 (2007).

17. P.T. Underhill, J.P. Hernandez-Ortiz, M.D. Graham, Phys. Rev. Lett. 100, 248101 (2008).

18. A. Baskaran, M.C. Marchetti, Proc. Natl. Acad. Sci. U.S.A. 106, 1556715572 (2009).

19. R.W. Nash, R. Adhikari, J. Tailleur, M.E. Cates, Phys. Rev. Lett. 104, 258101 (2010).

20. S. Rafaï, L. Jibuti, P. Peyla, Phys. Rev. Lett. 104, 098102 (2010).

21. F. Peruani, A. Deutsch, M. Bär, Phys. Rev. E 74, 030904 (2006).

22. J. Tailleur, M.E. Cates, Phys. Rev. Lett. 100, 218103 (2008).

23. F. Ginelli, F. Peruani, M. Bär, H. Chate, Phys. Rev. Lett. 104, 184502 (2010).

24. M. Enculescu, H. Stark, Phys. Rev. Lett. 107, 058301 (2011).

25. E.A. Keller, L.A. Segel, J. Theor. Biol. 30, 235 (1971).

26. J. Taktikos, V. Zaburdaev, H. Stark, Phys. Rev. E 85, 051901 (2012).

27. A. Pototsky, H. Stark, EPL 98, 50004 (2012).

28. R. Golestanian, Phys. Rev. Lett. 108, 038303 (2012).

29. J.S. Guasto, R. Rusconi, R. Stocker, Annu. Rev. Fluid. Mech. 44, 373 (2012).

30. S. Uppaluri, N. Heddergott, E. Stellamanns, S. Herminghaus, A. Zöttl, H. Stark, M. Engstler, T. Pfohl, Biophys. J. 103, 1162 (2012).

31. J.A. Riffell, R.K. Zimmer, J. Exp. Biol. 210, 3644 (2007).

32. F.P. Bretherton, L. Rothschild, Proc. R. Soc. London, Ser. B Biol. Sci. 153, 490 (1961). 
33. Marcos, H.C. Fu, T.R. Powers, R. Stocker, Proc. Natl. Acad. Sci. U.S.A 109, 4780 (2012).

34. W.M. Durham, J.O. Kessler, R. Stocker, Science 323, 1067 (2009).

35. O. Kessler, Nature 313, 218 (1985).

36. T.J. Pedley, J.O. Kessler, Proc. R. Soc. London, Ser. B 231, 47 (1987)

37. G. Zilman, J. Novak, Y. Benayahu, Mar. Biol. 154, 1 (2008).

38. A. Zöttl, H. Stark, Phys. Rev. Lett. 108, 218104 (2012).

39. C. Torney, Z. Neufeld, Phys. Rev. Lett. 99, 078101 (2007).

40. N. Khurana, J. Blawzdziewicz, N.T. Ouellette, Phys. Rev. Lett. 106, 198104 (2011).

41. F. Guzmán-Lastra, R. Soto, Phys. Rev. E 86, 037301 (2012).

42. R. Wittkowski, H. Löwen, Phys. Rev. E 85, 021406 (2012).

43. G.B. Jeffery, Proc. R. Soc. London, Ser. A 102, 161 (1922).
44. F.P. Bretherton, J. Fluid Mech. 14, 284 (1962).

45. J. Hill, O. Kalkanci, J.L. McMurry, H. Koser, Phys. Rev. Lett. 98, 068101 (2007).

46. A.P. Berke, L. Turner, H.C. Berg, E. Lauga, Phys. Rev. Lett. 101, 038102 (2008)

47. I. Llopis, I. Pagonabarraga, J. Non-Newton. Fluid. Mech. 165, 946 (2010).

48. K. Drescher, J. Dunkel, L.H. Cisneros, S. Ganguly, R.E. Goldstein, Proc. Natl. Acad. Sci. U.S.A 108, 10940 (2011).

49. G.L. Baker, Am. J. Phys. 74, 482 (2006).

50. P.G. Drazin, Nonlinear Systems (Cambridge University Press, Cambridge, 1994).

51. T.J. Pedley, J.O. Kessler, Annu. Rev. Fluid Mech. 24, 313 (1992).

52. P. Peyla, S. Rafaï, X. Garcia, Verhandl. DPG (VI) 47, 141 (2012). 